\title{
Regulation of AMPA Receptor Trafficking by Protein Ubiquitination
}

\author{
Jocelyn Widagdo, Sumasri Guntupalli, Se E. Jang and Victor Anggono* \\ Clem Jones Centre for Ageing Dementia Research, Queensland Brain Institute, The University of Queensland, Brisbane, \\ QLD, Australia
}

The molecular mechanisms underlying plastic changes in the strength and connectivity of excitatory synapses have been studied extensively for the past few decades and remain the most attractive cellular models of learning and memory. One of the major mechanisms that regulate synaptic plasticity is the dynamic adjustment of the $\alpha$-amino-3-hydroxy-5-methyl-4-isoxazolepropionic acid (AMPA)-type glutamate receptor content on the neuronal plasma membrane. The expression of surface AMPA receptors (AMPARs) is controlled by the delicate balance between the biosynthesis, dendritic transport, exocytosis, endocytosis, recycling and degradation of the receptors. These processes are dynamically regulated by AMPAR interacting proteins as well as by various post-translational modifications that occur on their cytoplasmic domains. In the last few years, protein ubiquitination has emerged as a major regulator of AMPAR intracellular trafficking. Dysregulation of AMPAR ubiquitination has also been implicated in the pathophysiology of Alzheimer's disease. Here we review recent advances in the field and provide insights into the role of protein ubiquitination in regulating AMPAR membrane trafficking and function. We also discuss how aberrant ubiquitination of AMPARs contributes to the pathogenesis of various neurological disorders, including Alzheimer's disease, chronic stress and epilepsy.

Edited by:

Argentina Lario Lago, University of California, San Francisco, United States

Reviewed by:

Ana Luisa Carvalho, University of Coimbra, Portugal Maria E. Rubio, University of Pittsburgh, United States

*Correspondence: Victor Anggono v.anggono@uq.edu.au

Received: 17 August 2017 Accepted: 12 October 2017 Published: 26 October 2017

Citation:

Widagdo J, Guntupalli S, Jang SE and Anggono V (2017) Regulation of AMPA Receptor Trafficking by Protein Ubiquitination.

Front. Mol. Neurosci. 10:347. doi: 10.3389/fnmol.2017.00347
Keywords: AMPA receptors, ubiquitin, E3 ligase, deubiquitinating enzyme (DUB), endosomal sorting, lysosome, protein degradation, synaptic plasticity

\section{INTRODUCTION}

The binding of glutamate to $\alpha$-amino-3-hydroxy-5-methyl-4-isoxazolepropionic acid (AMPA) receptors (AMPARs) mediates the fast, moment-to-moment transmission of excitatory signals in the mammalian central nervous system. The mammalian genome encodes four AMPAR subunits, GluA1-4. These combine as a dimer of dimers to form functional tetramers that are generally permeable only to $\mathrm{Na}^{+}$and $\mathrm{K}^{+}$ions, with the exception of GluA2-lacking AMPARs, which also conduct additional $\mathrm{Ca}^{2+}$ ions into dendritic spines (Sukumaran et al., 2012). AMPARs cycle into and out of the neuronal plasma membrane under basal conditions, and these trafficking patterns can be rapidly and dynamically modulated in an activity-dependent manner. Dynamic trafficking of AMPARs is one of the major mechanisms underpinning various forms of synaptic plasticity, including Hebbian and homeostatic plasticity (Pozo and Goda, 2010; Huganir and Nicoll, 2013). These processes are tightly regulated by the orchestrated binding of AMPAR binding proteins, as well as by reversible post-translational modifications that occur on the carboxyl terminal domains of AMPAR subunits (Anggono and Huganir, 2012; Lu and Roche, 2012).

Ubiquitination is a reversible post-translational modification that regulates a myriad of physiological processes, including protein degradation, endocytosis and the sorting and trafficking of transmembrane proteins (Hershko and Ciechanover, 1998). It involves the covalent 
attachment of a highly conserved 76 amino acid ubiquitin moiety (monoubiquitination) or polymeric ubiquitin chains (polyubiquitination) to a lysine residue of a substrate protein (Hershko and Ciechanover, 1998). The conjugation of ubiquitin to a substrate depends on an enzymatic cascade that comprises ubiquitin-activating enzymes (E1s), ubiquitin-conjugating enzymes (E2s) and ubiquitin ligases (E3s). Although the ubiquitin pathway is important in all cells, it is now clear that this pathway subserves a range of important functions in neurons and is necessary for learning and memory (Mabb and Ehlers, 2010). Importantly, the ubiquitin-proteasome system is crucial in regulating AMPAR trafficking and turnover (Patrick et al., 2003; Zhang et al., 2009; Yuen et al., 2012). The evidence demonstrating the ubiquitination of the glutamate receptor, GLR-1 and its role in regulating receptor abundance was first obtained in the nematode C. elegans (Burbea et al., 2002). Since then, several studies have also reported the ubiquitination of AMPARs in mammalian neurons (Schwarz et al., 2010; Fu et al., 2011; Lin et al., 2011; Lussier et al., 2011; Widagdo et al., 2015). In this review, we highlight recent progress in the field that advances our understanding of the molecular regulation of AMPAR function by protein ubiquitination and its potential implication in the treatment of various disorders, including Alzheimer's disease, epilepsy and chronic stress. Given the limited knowledge on the regulation of the GluA3 and GluA4 subunits of AMPARs by post-translational ubiquitination, this review focuses only on GluA1 and GluA2, the heteromers of which constitute the majority of AMPARs in the forebrain.

\section{MECHANISMS UNDERLYING THE UBIQUITINATION OF AMPARS}

\section{Ubiquitination of AMPARs Is $\mathrm{Ca}^{2+}$-Dependent}

The ubiquitination of AMPARs is initially triggered by the binding of ligand to the receptors. All four subunits of AMPARs, GluA1-4, undergo activity-dependent ubiquitination when neurons are stimulated with AMPA or bicuculline (Schwarz et al., 2010; Lussier et al., 2011; Widagdo et al., 2015). As a selective $\mathrm{GABA}_{\mathrm{A}}$ receptor antagonist, bicuculline enhances the release of glutamate from the presynaptic terminals and therefore preferentially activates AMPARs that are located at the synapse, while bath application of AMPA co-activates both synaptic and extrasynaptic receptors. The major ubiquitination site for the GluA1 subunit has been mapped to Lys-868 located in the distal C-terminal tail of the receptor, whereas GluA2 is predominantly ubiquitinated at Lys-870 and Lys-882 in neurons (Figure 1; Widagdo et al., 2015).

In addition to ligand binding to AMPARs, subsequent depolarization of the postsynaptic membrane is also required for AMPAR ubiquitination. This allows the second messenger $\mathrm{Ca}^{2+}$ to enter the postsynaptic compartment through L-type voltage-gated $\mathrm{Ca}^{2+}$ channels (L-VGCCs) and activates a $\mathrm{Ca}^{2+}$-dependent signaling cascade that involves the activation of $\mathrm{Ca}^{2+} /$ calmodulin-dependent kinase II (CaMKII; Lussier et al., 2011; Widagdo et al., 2015). Mechanistically, the role of CaMKII in regulating AMPAR ubiquitination is currently unknown, but CaMKII is presumably involved in direct phosphorylation and/or activation of E3 ligase(s) for AMPAR subunits. Interestingly, neither agonist-induced activation of NMDA receptors (NMDARs) nor NMDAR-dependent signaling is required for AMPA-induced ubiquitination of AMPARs (Schwarz et al., 2010; Lussier et al., 2011; Widagdo et al., 2015). However, the activity of NMDARs plays an important role in modulating the ubiquitination of AMPARs induced by bicuculline (Lussier et al., 2011; Widagdo et al., 2015). These findings suggest that AMPA and bicuculline stimulate two molecularly distinct signaling pathways, which may result in the recruitment of different E3 ligases and dictate the routes of AMPAR trafficking and subsequent degradation through the lysosomal or proteasomal pathways.

\section{E3 Ligases for AMPARs}

To date, four different E3 ligases, namely neural precursor cellexpressed developmentally downregulated gene 4-1 (Nedd4-1), Nedd4-2, RNF167 and APC ${ }^{\text {Cdh1 }}$, have been shown to mediate the ubiquitination of AMPARs in mammalian central neurons under different stimulation conditions (Schwarz et al., 2010; Fu et al., 2011; Lin et al., 2011; Lussier et al., 2012; Zhu et al., 2017).

\section{Nedd4}

The Nedd4 family of E3 ligases is characterized by the presence of the homologous to E6-AP C-terminus (HECT) domain, which first accepts ubiquitin from an E2 ligase onto its catalytic cysteine residue, prior to transferring it to the substrate (Scheffner and Kumar, 2014). The human genome encodes nine members of the Nedd4 E3 ligase family, each of which contains an N-terminal $\mathrm{C} 2 \mathrm{Ca}^{2+}$ /phospholipid-binding domain, multiple WW protein-protein interaction domains and a C-terminal HECT domain. These ligases preferentially form K63-linked polyubiquitin chains on their substrates (Kim et al., 2007). Nedd4-1 was the first E3 ligase which was shown to interact with and facilitate the ubiquitination of GluA1 in neurons (Schwarz et al., 2010). Overexpression of Nedd4-1 reduces surface AMPARs due to enhanced endocytosis and the accumulation of internalized GluA1 in late endosomes (Schwarz et al., 2010; Lin et al., 2011; Scudder et al., 2014). Interestingly, Nedd4-1 is rapidly redistributed to synapses upon AMPAR activation (Hou et al., 2011; Scudder et al., 2014). This activity-dependent localization of Nedd4-1 requires the functional C2 domain (Scudder et al., 2014), presumably to mediate $\mathrm{Ca}^{2+}$-dependent binding to phospholipids (Plant et al., 1997). Furthermore, overexpression of Nedd4-1 with deletion of the $\mathrm{C} 2$ domain fails to cause any reduction in synaptic AMPARs, suggesting the importance of Nedd4-1 $\mathrm{Ca}^{2+}$. binding in regulating GluA1 surface expression (Scudder et al., 2014).

More recently, another closely related E3 ligase, Nedd4-2 (also known as Nedd4-like) has also been demonstrated to facilitate GluA1 ubiquitination in neurons (Jewett et al., 2015; Zhu et al., 2017). Nedd4-2, when bound to the adaptor protein $14-3-3 \varepsilon$, can 


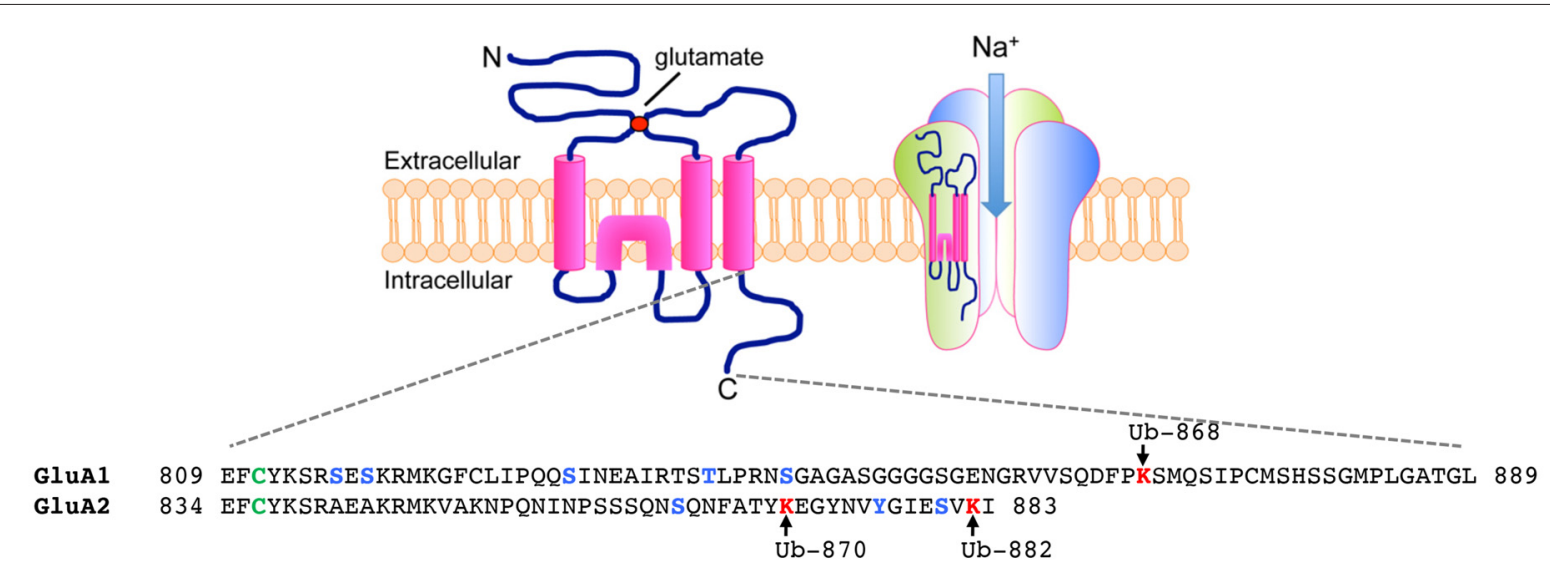

FIGURE 1 | Ubiquitination of $\alpha$-amino-3-hydroxy-5-methyl-4-isoxazolepropionic acid receptors (AMPARs) on the C-terminal of GluA1 and GluA2 subunits. (Top) Each AMPAR subunit is composed of an extracellular glutamate binding region, four transmembrane domains, two intracellular loops and an intracellular carboxy-tail. Four subunits (two homodimers) are assembled into a functional tetramer that is permeable to $\mathrm{Na}^{+}$ions. (Bottom) Amino acid sequences of the carboxy-tails of GluA1 and GluA2 showing sites of post-translational ubiquitination (lysines in red, arrows), phosphorylation (serines, threonines and tyrosine in blue) and palmitoylation (cysteines in green).

directly ubiquitinate GluA1 in an in vitro ubiquitination assay (Zhu et al., 2017). Loss of Nedd4-2 function inhibits picrotoxininduced ubiquitination of GluA1 in primary neurons (Jewett et al., 2015). As a consequence, the expression of GluA1 is elevated in the brain of the seizure prone Nedd4- $2^{\text {andi }}$ mouse, in which the major isoform of Nedd4-2 is deleted (Zhu et al., 2017).

Although Nedd4- 1 and Nedd4-2 are structurally very similar, they appear to have distinct cellular functions and target specific proteins for ubiquitination (Yang and Kumar, 2010). Many of the identified Nedd4-1 substrates are receptor tyrosine kinases, whereas Nedd4-2 preferentially targets ion channels (Persaud et al., 2009). However, Nedd4-1 and Nedd4-2 also share several common substrates, which are likely to include GluA1 in the brain. It is plausible that the recruitment of these two E3 ligases might be regulated by different types of neuronal stimulation, providing selectivity and specificity in the regulation of AMPAR trafficking and function.

\section{RNF167}

RING finger protein (RNF) 167 is a member of the really interesting new gene (RING) E3 ligase family that comprises over 600 proteins in the human genome. Unlike the HECT domain, the RING domain lacks a catalytic cysteine and merely acts as a scaffold to facilitate the direct transfer of ubiquitin from E2 to the substrate. RNF167 is an integral membrane protein that is localized to the plasma membrane, endosomes and lysosomes, and has a role in regulating endosomal trafficking and the degradation of substrates in lysosomes (Lussier et al., 2012; Yamazaki et al., 2013; van Dijk et al., 2014; Deshar et al., 2016). It was first identified as an $E 3$ ligase for the GluA2 subunit of AMPARs (Lussier et al., 2012). shRNA-mediated knockdown of RNF167 significantly reduces GluA2/3 ubiquitination in neurons following bicuculline stimulation. Interestingly, loss of RNF167 function not only enhances the expression of surface GluA2/3, but also upregulates surface GluA1 levels in cortical neurons. This suggests that RNF167 could be another E3 ligase for GluA1, although this has not been demonstrated biochemically. In addition, whether RNF167 is specifically recruited during heightened synaptic activity to ubiqutinate only the GluA2 and GluA3 subunits is yet to be determined.

\section{APC Cdh1}

Anaphase promoting complex (APC) is a large multisubunit E3 ligase that targets key cell cycle regulatory proteins for proteasomal degradation (Huang and Bonni, 2016). The catalytic core consists of two subunits, namely the APC2 scaffold protein and the RING domain-containing APC11 (Harper et al., 2002). Cdh1 represents the major regulator and activator of APC in mature neurons and recognizes its substrates via various peptide motifs, including the D (destruction), $\mathrm{A}$ and KEN boxes. $\mathrm{APC}^{\mathrm{Cdh} 1}$ plays critical roles in brain development, dendritic integrity, synaptic plasticity, learning and memory (Huang and Bonni, 2016). APC was first shown to regulate the abundance of GLR-1 in C. elegans; however no biochemical evidence was presented to suggest a role for APC as the GLR-1 E3 ligase (Juo and Kaplan, 2004). In mammalian neurons, APC Cdh1 interacts with the EphA4 receptor to mediate the ephrin-induced proteasomal degradation of AMPARs (Fu et al., 2011). Fu et al. (2011) further demonstrated the ability of APC $^{\text {Cdh1 } 1}$ to ubiquitinate GluA1 in HEK293 cells, a process that is dependent on the binding of the Cdh1 WD40 domain to the D box motifs located within the GluAl extracellular $\mathrm{N}$-terminal domain. Furthermore, mutation of all four lysine residues in the GluA1 C-terminal tail does not inhibit APC ${ }^{\text {Cdh1 }}$ mediated ubiquitination and degradation in HEK293 cells. Given these facts, we speculate that $\mathrm{APC}^{\mathrm{Cdh} 1}$-mediated ubiquitination of GluAl may be the result of an overexpression artifact in HEK293 cells, potentially through the activation of the endoplasmic reticulum-associated degradation pathway. It is therefore critical to examine the effect of Cdh1 loss of 
function on activity-induced AMPAR ubiquitination in neurons. Notwithstanding the clear physiological importance of APC ${ }^{\mathrm{Cdh} 1}$ in synaptic plasticity, learning and memory, we propose that $\mathrm{APC}^{\mathrm{Cdh} 1}$ may regulate the trafficking of AMPARs through the ubiquitination of AMPAR-related postsynaptic scaffold proteins or other neuronal substrates, including the recently reported Fragile X-associated protein FMRP and the microtubule regulator Rock2 (Huang et al., 2015; Bobo-Jimenéz et al., 2017).

\section{Deubiquitinating Enzyme for AMPARs}

Deubiquitinating enzymes (DUBs) catalyze the removal of covalently attached ubiquitin from proteins, thereby controlling ubiquitin signaling and maintaining the free ubiquitin pool in cells. In contrast to the diversity of ubiquitin E3 ligase, the human genome encodes only 95 DUBs (Sowa et al., 2009), two of which have been shown to deubiquitinate AMPARs (Scudder et al., 2014; Huo et al., 2015).

\section{USP8}

The synapse-enriched (DUB), ubiquitin-specific protease 8 (USP8) is a key regulator of the endosomal sorting complex required for transport (ESCRT) pathway that is essential for the formation of multivesicular bodies and lysosomes (Wright et al., 2011; Lee and Gao, 2012). Overexpression of USP8 in cultured neurons reduces the level of agonistinduced AMPAR ubiquitination, leading to increases in the number of surface GluA1 and synaptic AMPARs (Scudder et al., 2014). These effects are abrogated by a mutation of the catalytic cysteine residue in the USP domain, indicating the critical requirement of USP8 deubiquitinating activity in regulating AMPAR trafficking. Furthermore, shRNA-mediated knockdown of USP8 is sufficient to enhance the basal level of AMPAR ubiquitination in primary neurons. The expression and activity of USP8 are regulated by neuronal activity. The activation of NMDARs induces $\mathrm{Ca}^{2+}$-dependent dephosphorylation of USP8 by a still unknown tyrosine phosphatase, thereby enhancing its catalytic activity (Scudder et al., 2014).

\section{USP46}

USP46 was first identified as a DUB that regulates the level of GLR-1 in the ventral nerve cord of C. elegans (Kowalski et al., 2011), and was subsequently shown to regulate AMPAR trafficking in mammalian neurons (Huo et al., 2015). USP46 is enriched at synapses and expressed throughout all brain regions. Similar to USP8, knockdown of USP46 also enhances basal GluA1 ubiquitination and reduces its expression in neurons. Congruent with its role as a GluA1 DUB, overexpression of USP46 reduces GluA1 internalization, leading to an increase in the level of surface AMPARs.

\section{Cross-Talk of AMPAR Phosphorylation and Ubiquitination}

In addition to ubiquitination, AMPAR subunits are also subjected to various reversible post-translational modifications, including phosphorylation, palmitoylation and nitrosylation
(Roche et al., 1996; Hayashi et al., 2009; Selvakumar et al., 2013). These different post-translational modifications can functionally interact to provide extra layers of fine regulation and modulation of AMPAR trafficking and function (Lu and Roche, 2012). For example, the palmitoylation of the GluA1 subunit at Cys-811 modulates protein kinase C (PKC)-dependent phosphorylation at Ser-816/818, thereby contributing to the regulation of AMPAR insertion into the plasma membrane and synaptic plasticity (Lin et al., 2009). More recently, we demonstrated a cross-talk between GluA1 phosphorylation and ubiquitination. Inhibition of GluA1 ubiquitination causes enhanced protein kinase A (PKA) phosphorylation at Ser-845, but has no effect on PKC/CaMKII phosphorylation at Ser-831 (Guntupalli et al., 2017). Interestingly, the phosphomimetic S845D mutant negatively regulates GluA1 ubiquitination by reducing its ability to interact with Nedd4-1. Such a dynamic cross-modulation of GluA1 ubiquitination and phosphorylation is critical for the membrane sorting of AMPARs, that ultimately determines the number of receptors on the plasma membrane. It remains to be determined whether the ubiquitination of AMPARs causes cross-talk with other post-translational modifications under specific neuronal states.

\section{FUNCTIONAL ROLES OF AMPAR UBIQUITINATION}

For historical reasons, ubiquitination has always been associated with protein degradation. However, new roles of ubiquitin in gene transcription, apoptosis, cell cycle, the DNA damage response, cell signaling, protein localization and trafficking have emerged in recent years (Ikeda and Dikic, 2008). The diversity of ubiquitin function is brought about in large part by the ability of ubiquitin to form a polyubiquitin chain that is conjugated on one of its seven lysine residues (Lys6, Lys-11, Lys-27, Lys-29, Lys-33, Lys-48 and Lys-63) that adopt various structural conformations, thereby creating a range of different molecular signals in the cell (Komander and Rape, 2012). For example, K48-linked polyubiquitination commonly leads proteins towards proteasomal degradation. In contrast, K63-linked substrates often undergo non-proteasomal fates, including protein endocytosis, sorting and receptor trafficking.

\section{AMPAR Trafficking}

The GluA1 and GluA2 subunits of AMPARs are modified by K63-linked polyubiquitin chains when neurons are stimulated with the agonist AMPA in the presence of the NMDAR antagonist, D-APV (Widagdo et al., 2015). It is also well established that the activation of AMPARs in the absence of NMDAR activity induces AMPAR internalization and sorting towards late endosomes for degradation by lysosomes (Ehlers, 2000). Together with the fact that only surface receptors undergo agonist-induced ubiquitination (Widagdo et al., 2015), it is therefore plausible that the ubiquitination of AMPARs is involved in both the endocytosis and post-endocytic sorting 
of these receptors towards late endosomes. While data from multiple labs have lent support to the role of protein ubiquitination in regulating the intracellular trafficking of AMPARs to late endosomes (Schwarz et al., 2010; Widagdo et al., 2015), the cellular function of GluA1 and GluA2 ubiquitination in mediating AMPAR endocytosis remains controversial (Goo et al., 2015; Widagdo and Anggono, 2015).

There are several pieces of evidence which support the idea that ubiquitination of GluA1 acts as an endocytic signal for AMPARs. These include: (a) overexpression of Nedd4-1 in neurons, which enhances GluAl ubiquitination, causes a reduction in the number of AMPARs on the plasma membrane (Schwarz et al., 2010; Lin et al., 2011); (b) shRNA-mediated knockdown of Nedd4-1 increases the rate of GluA1 internalization (Schwarz et al., 2010); (c) overexpression of USP46, which downregulates GluA1 ubiquitination, decreases the accumulation of internalized GluA1 in neurons (Huo et al., 2015); and (d) overexpression of GluA1-4KR ubiquitindeficient mutants (where all four C-terminal lysine residues have been substituted with arginines) blocks AMPA-induced GluA1 internalization (Schwarz et al., 2010; Lin et al., 2011). Moreover, the endocytic adaptor Eps15 (epidermal growth factor receptor substrate 15) has been reported to interact with GluA1 and facilitate AMPAR internalization (Lin and Man, 2014). Interestingly, this interaction is dependent on GluA1 ubiquitination and Eps15 C-terminal ubiquitin interacting motifs. Together, these data support the notion that ubiquitination of AMPARs is necessary for and occurs prior to receptor internalization.

On the other hand, there are two studies that argue against the role of ubiquitin in mediating AMPAR endocytosis (Lussier et al., 2011; Widagdo et al., 2015). In these studies, the authors used small molecule inhibitors of dynamin, an enzyme that is essential for membrane fission during endocytosis, namely dynasore and dynole (Macia et al., 2006; Hill et al., 2009), as well as sucrose to inhibit the formation of clathrin-coated pits, and found that these treatments prevent AMPA- or bicucullineinduced ubiquitination of all AMPAR subunits in cultured neurons. This suggests that endocytosis precedes the conjugation of ubiquitin moieties to AMPARs. Indeed, we found that the same GluA1-4KR ubiquitin-deficient mutant does not affect either the surface expression or agonist-induced internalization of AMPARs (Widagdo et al., 2015), in contrast to the results of previous studies (Schwarz et al., 2010; Lin et al., 2011). Instead, we observed that the GluA1-4KR mutant is mis-sorted into recycling endosomes and returns to the plasma membrane. Interestingly, direct ubiquitination of several transmembrane receptors from the receptor tyrosine kinase and cytokine receptor superfamilies, including the epidermal growth factor receptor, the fibroblast growth factor receptor and the growth factor receptor, is not required for their internalization (Govers et al., 1999; Huang et al., 2007; Haugsten et al., 2008).

The discrepancies found in these studies could arise due to differences in experimental conditions. These may include the duration and intensity of neuronal stimulation, as well as the level of AMPAR subunit overexpression, which could alter the subunit composition of the surface receptors being examined. Given that AMPARs are known to undergo distinct endocytic pathways (Beattie et al., 2000; Ehlers, 2000; Lin et al., 2000), the differential requirement of ubiquitin in regulating the internalization of AMPARs cannot be ruled out. Notwithstanding the debatable role of AMPAR ubiquitination in receptor endocytosis, the consensus is that ubiquitination of AMPARs controls the intracellular sorting of receptors to late endosomes for degradation in lysosomes.

\section{Synaptic Plasticity}

The dynamic trafficking and number of AMPARs on the neuronal plasma membrane are the major determinants of the plasticity of excitatory synapses (Huganir and Nicoll, 2013). The mechanisms that control the endosomal sorting and trafficking of AMPARs are involved in both Hebbian and homeostatic plasticity. For example, protein interacting with C-kinase 1 (PICK1), which directly interacts with GluA2/3 subunits and regulates the endosomal recycling of AMPARs (Xia et al., 1999; Citri et al., 2010; Widagdo et al., 2016), is a critical regulator of long-term potentiation (LTP), long-term depression (LTD) and homeostatic synaptic scaling (Terashima et al., 2008; Thorsen et al., 2010; Anggono et al., 2011). Furthermore, the balance between Rab7- and Rab11-dependent recycling or trafficking of AMPARs towards late endosomes has also been shown to determine the outcome of LTD, underscoring the importance of membrane sorting decisions in synaptic plasticity (Fernández-Monreal et al., 2012). Although there is currently no empirical evidence that demonstrates the role of AMPAR ubiquitination in controlling Hebbian plasticity, there have been several studies that show the involvement of GluA1/2 ubiquitination in homeostatic synaptic scaling (Fu et al., 2011; Hou et al., 2011; Scudder et al., 2014; Jewett et al., 2015).

During prolonged alteration in synaptic activity, homeostatic plasticity maintains neuronal stability by adjusting synaptic properties, including the number of synaptic AMPARs, in order to keep neuronal excitability close to the internal target firing range (Turrigiano, 2012). Downscaling of AMPAR-mediated synaptic currents in primary neurons can be observed during chronic elevation of synaptic activity induced by the blockade of $\mathrm{GABA}_{\mathrm{A}}$ receptors using bicuculline or picrotoxin (O'Brien et al., 1998; Turrigiano et al., 1998). More recently, homeostatic scaling-down of excitatory synapses has also been observed in vivo during sleep, a process that is essential for memory consolidation (Diering et al., 2017).

Recent studies have demonstrated the involvement of Nedd4-1 and Nedd4-2 in mediating homeostatic synaptic downscaling of AMPARs in cultured neurons either by lightcontrolled single synaptic activation (Hou et al., 2011), or by the application of $\mathrm{GABA}_{\mathrm{A}}$ receptor antagonists (Scudder et al., 2014; Jewett et al., 2015). Under these conditions, the levels of AMPAR ubiquitination are significantly upregulated concomitant with the reduction of total AMPAR expression in neurons. In one study, Jewett et al. (2015) reported that the transcription of Nedd4-2 mRNA and Nedd4-2 protein are specifically upregulated following chronic elevation of 
neuronal activity (Jewett et al., 2015). The detailed mechanisms of picrotoxin-induced increase in Nedd4-2 expression is not well understood, but it involves the Akt-Mdm2-p53 signaling pathway. In contrast, a separate study by Scudder et al. (2014) reported that Nedd4-1 expression could indeed be upregulated following chronic bicuculline treatment. Moreover, they also found that the expression of USP8 was downregulated, further promoting the ubiquitination of AMPARs. Another mechanism that has been shown to control the reduction of AMPAR-mediated synaptic strength involves the $\mathrm{APC}^{\mathrm{Cdh} 1}$-dependent proteasome pathway (Fu et al., 2011). However, the evidence for direct involvement of $\mathrm{APC}^{\mathrm{Cdh} 1}$ in mediating AMPAR ubiquitination and degradation is inconclusive.

\section{AMPAR UBIQUITINATION IN NEUROLOGICAL DISORDERS}

Homeostatic regulation of the number of ion channels and transmembrane receptors on the plasma membrane is largely achieved by endocytosis mechanisms and downstream endosomal trafficking. Perturbations of AMPAR trafficking have been implicated in a range of neurological disorders. For examples, neuronal hyperactivity due to gain of AMPAR function can lead to epileptic seizures, whereas AMPAR hypofunction is associated with synaptic depression that is commonly linked to disorders such as schizophrenia, chronic stress and Alzheimer's disease. Recent evidence has started to shed light on the involvement of ubiquitin-mediated trafficking of AMPARs in the pathophysiology of these disorders.

\section{Alzheimer's Disease}

Early memory deficits and progressive loss of higher cognitive functions are common clinical features of Alzheimer's disease, which is characterized by the presence of insoluble aggregates of extracellular amyloid-beta $(\mathrm{A} \beta)$ peptides and intracellular filaments composed of hyperphosphorylated tau. Strong evidence from human genetics and transgenic mouse models has indicated a role for $A \beta$ in the etiology and pathogenesis of Alzheimer's disease (Selkoe, 2002). It is well established that soluble oligomeric forms of $\mathrm{A} \beta$ peptides exert strong detrimental effects on the structure and functional state of synapses, in part by promoting the internalization and degradation of AMPARs that eventually lead to synaptic depression (Sheng et al., 2012; Guntupalli et al., 2016). In addition to $A \beta$, a newly identified cleavage product of amyloid precursor protein that is enriched in the dystrophic neurites in an Alzheimer's disease mouse model and in post-mortem Alzheimer's brains, termed $A \eta$ peptide, has also been shown to impair excitatory synaptic transmission and hippocampal LTP (Willem et al., 2015).

Recent work from our own and the Patrick laboratory have directly demonstrated the direct involvement of AMPAR ubiquitination as a critical pathway in mediating the $A \beta$-induced synaptic depression in neurons (Rodrigues et al., 2016;
Guntupalli et al., 2017). Acute exposure of cultured neurons to soluble $A \beta$ oligomers induces AMPAR ubiquitination concomitant with the removal of the receptors from the plasma membrane (Guntupalli et al., 2017). Importantly, expression of GluA1-K868R or GluA1-4KR ubiquitin-deficient mutants inhibits the adverse effects of $A \beta$ on the surface expression of AMPARs in neurons. Prolonged exposure of neurons to the supernatant of 7PA2 Chinese hamster ovary cells, which naturally secrete A $\eta$ peptides (Willem et al., 2015), also enhances AMPAR ubiqutination and consequently causes a reduction in AMPAR-mediated currents and spine loss (Rodrigues et al., 2016). These effects can be rescued by knocking down Nedd4-1 expression, suggesting that the activity of Nedd4- 1 is necessary for $A \eta$-induced synaptic alterations in neurons. Given that the expression of Nedd4-1 is upregulated in the human Alzheimer's brain, a small molecule inhibitor of Nedd4-1 may represent a possible therapeutic for reversing synaptic depression and associated cognitive impairments in patients with this disease.

\section{Epilepsy}

Epilepsy is a chronic neurological disorder characterized by recurring and unprovoked seizures that arise from abnormally synchronous neuronal network activity in a focal area or throughout the entire brain. Genetic or acquired ion channel dysfunctions that alter the excitation and inhibition balance of synaptic connectivity underlie epileptiform discharges. Studies have shown that the inhibition of AMPAR-mediated neuronal excitation confers seizure protection in a broad range of animal seizure models (Rogawski, 2013). Indeed, a highly potent non-competitive AMPAR antagonist, perampanel, has been used clinically to treat patients with partial-onset and tonic-clonic seizures (French et al., 2012, 2015).

Genetic studies have identified at least three different mis-sense mutations in the Nedd4-2 gene in patients with epilepsy (Dibbens et al., 2007; Epi4K Consortium et al., 2013; Vanli-Yavuz et al., 2015). These mutations disrupt Nedd4-2 binding to $14-3-3 \varepsilon$, thereby reducing its ability to ubiquitinate GluA1 (Zhu et al., 2017). This explains the apparent elevation in seizure susceptibility in the Nedd4- $2^{\text {andi }}$ mouse, in which the major isoform of Nedd4-2 is selectively deficient in the brain. Remarkably, genetically reducing the GluA1 level by crossing Nedd4-2 andi mice with $G l u A 1^{+/-}$ heterozygous mice normalizes this effect, underscoring the importance of AMPAR ubiquitination in maintaining the optimal balance of neuronal excitation and inhibition in the brain.

\section{Chronic Stress}

Stress induces the release of glucocorticoids that alter glutamatergic neurotransmission and synaptic plasticity, which could subsequently trigger maladaptive changes in cognitive function (Popoli et al., 2012). It has been shown that repeated stress impairs glutamatergic transmission in the principal neurons in the prefrontal cortex of juvenile male rats (Yuen et al., 2012). One of the underlying mechanisms 


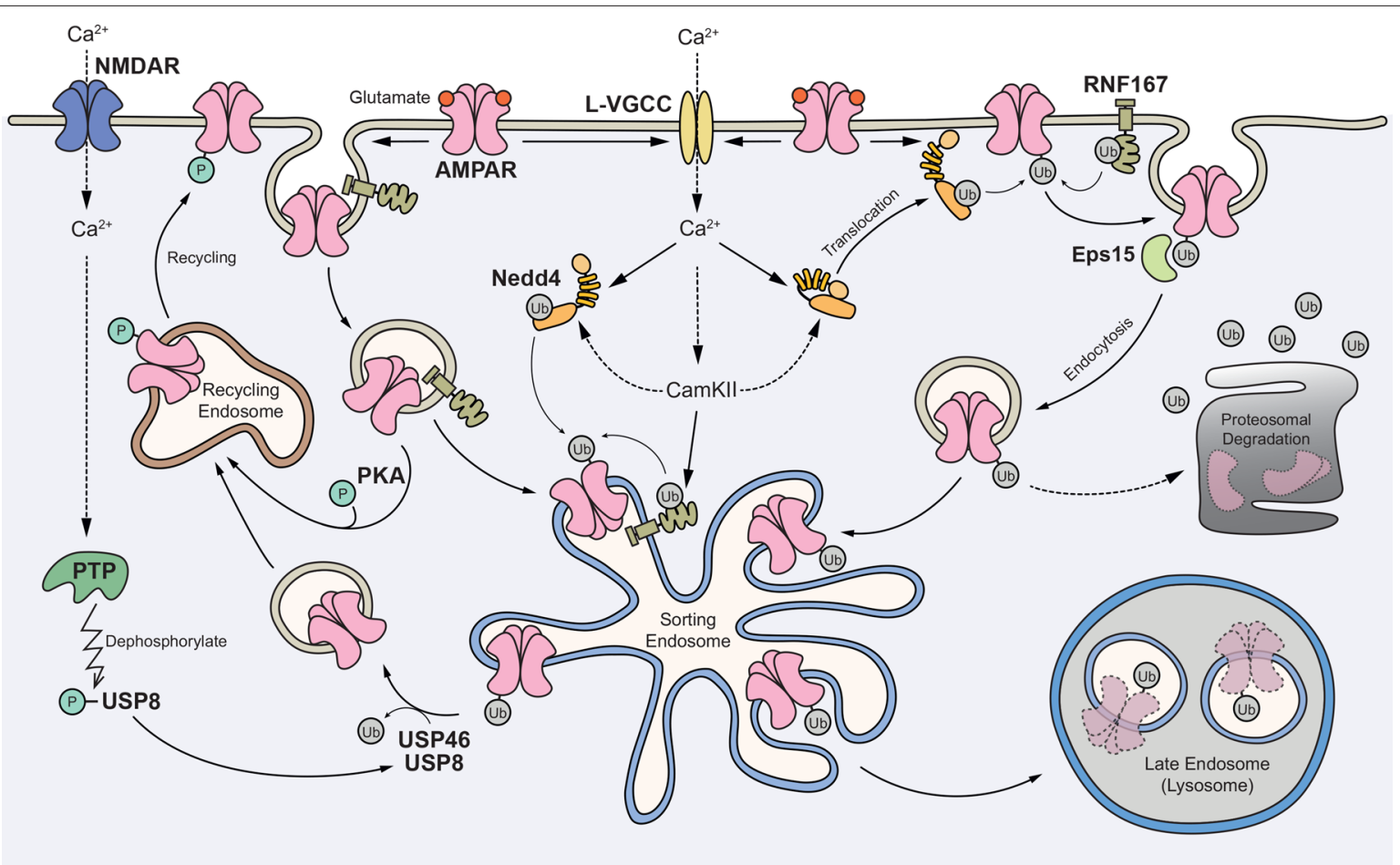

FIGURE 2 | Proposed model for the role of AMPAR ubiquitination in regulating receptor trafficking and degradation. Glutamate binding to AMPARs mediates depolarization of the postsynaptic membrane and the opening of L-type voltage-gated $\mathrm{Ca}^{2+}$ channels (L-VGCCs). The rise in intracellular Ca ${ }^{2+}$ subsequently activates E3 ligases through $\mathrm{Ca}^{2+}$-dependent translocation of neural precursor cell-expressed developmentally downregulated gene 4-1 (Nedd4-1) to the plasma membrane and/or direct phosphorylation of Nedd4 and RNF167 by Ca ${ }^{2+}$ /calmodulin-dependent kinase II (CaMKII). In one scheme, surface AMPARs are internalized without ubiquitination but are subsequently ubiquitinated in the endosomes. In another scheme, ubiquitination of surface receptors recruits the binding of an endocytic adaptor Eps15 and facilitates the internalization of AMPARs. Ubiquitinated AMPARs are sorted to late endosomes and degraded in lysosomes. The activation of NMDARs can recruit USP8, and potentially USP46, to deubiquitinate AMPARs and promote their recycling back to the plasma membrane. Through an unknown mechanism, ubiquitinated AMPARs may also be degraded through the proteasome system.

involves a glucocorticoid receptor-dependent reduction in AMPAR- and NMDAR-mediated synaptic transmission due to enhancement of ubiquitination and proteasomal degradation of GluA1 and GluN1 subunits, respectively. Importantly, shRNA-mediated knockdown of Nedd4-1 and Fbx2 (the E3 ligase for GluN1) in the prefrontal cortex prevents the loss of glutamatergic responses and is able to rescue cognitive deficits in stressed animals. These data further underscore the important roles of AMPAR ubiquitination signaling in mediating synaptic depression under pathological conditions.

\section{CONCLUDING REMARKS}

Although studies that emerged in the last few years have demonstrated an important function of the ubiquitin signaling pathway in regulating AMPAR trafficking and function, this field of research is still in its infancy. The roles of AMPAR ubiquitination in controlling receptor endocytosis and degradation (proteasomal vs. lysosomal) are controversial and debatable (Goo et al., 2015; Widagdo and Anggono, 2015), owing to differences in experimental systems, including but not limited to the strength and duration of neuronal stimulation, as well as the model system (primary neurons vs. heterologous cells). Given that AMPARs are not typically present in HEK293 cells, overexpression of any subunit of AMPARs may trigger the cellular stress response and ubiquitinmediated protein degradation. For this reason, studies of AMPAR ubiquitination in heterologous systems, such as those performed in HEK293 cells, are not ideal and must be interpreted with caution.

In summary, we propose the following working model (Figure 2). First, glutamate-mediated activation of AMPARs depolarizes the postsynaptic membrane, leading to the opening of L-VGCCs. The rise in intracellular $\mathrm{Ca}^{2+}$ subsequently activates E3 ligases through $\mathrm{Ca}^{2+}$-dependent translocation of Nedd4-1 to the plasma membrane and/or direct phosphorylation of Nedd4 and RNF167 by CaMKII. In one scheme, surface AMPARs are internalized without ubiquitination but are subsequently ubiquitinated in endosomes. In another scheme, ubiquitination of surface receptors recruits the binding of an endocytic adaptor, Eps15 and facilitates the internalization of AMPARs. Ubiquitinated AMPARs are then sorted to late endosomes and degraded in lysosomes. The activation of NMDARs can recruit USP8, and potentially USP46, to deubiquitinate AMPARs and promote their recycling back 
to the plasma membrane. Through an unknown mechanism, ubiquitinated AMPARs may also be degraded through the proteasome system.

Similar to kinases, components of the ubiquitination systems are often dysregulated in disease. Several recent findings have started to implicate dysregulation of AMPAR ubiquitination in the pathophysiology of Alzheimer's disease, epilepsy and chronic stress (Yuen et al., 2012; Rodrigues et al., 2016; Guntupalli et al., 2017; Zhu et al., 2017). Given the importance of ubiquitination in regulating the fate of receptors, inhibition of E3 ligases and/or DUBs may provide a possible therapeutic mechanism by restoring receptor function (Huang and Dixit, 2016). Future studies should focus on the detailed mechanisms that regulate stimulus- and subunit-specific regulation of AMPAR subunits in neurons during physiological and pathological conditions.

\section{REFERENCES}

Anggono, V., and Huganir, R. L. (2012). Regulation of AMPA receptor trafficking and synaptic plasticity. Curr. Opin. Neurobiol. 22, 461-469. doi: 10.1016/j.conb. 2011.12.006

Anggono, V., Clem, R. L., and Huganir, R. L. (2011). PICK1 loss of function occludes homeostatic synaptic scaling. J. Neurosci. 31, 2188-2196. doi: 10.1523/JNEUROSCI.5633-10.2011

Beattie, E. C., Carroll, R. C., Yu, X., Morishita, W., Yasuda, H., von Zastrow, M., et al. (2000). Regulation of AMPA receptor endocytosis by a signaling mechanism shared with LTD. Nat. Neurosci. 3, 1291-1300. doi: 10.1038/ 81823

Bobo-Jimenéz, V., Delgado-Esteban, M., Angibaud, J., Sánchez-Morán, I., de la Fuente, A., Yajeya, J., et al. (2017). APC/C $\mathrm{C}^{\mathrm{Cdh} 1}$-Rock2 pathway controls dendritic integrity and memory. Proc. Natl. Acad. Sci. U S A 114, 4513-4518. doi: 10.1073/pnas.1616024114

Burbea, M., Dreier, L., Dittman, J. S., Grunwald, M. E., and Kaplan, J. M. (2002). Ubiquitin and AP180 regulate the abundance of GLR-1 glutamate receptors at postsynaptic elements in C. elegans. Neuron 35, 107-120. doi: 10.1016/s08966273(02)00749-3

Citri, A., Bhattacharyya, S., Ma, C., Morishita, W., Fang, S., Rizo, J., et al. (2010). Calcium binding to PICK1 is essential for the intracellular retention of AMPA receptors underlying long-term depression. J. Neurosci. 30, 16437-16452. doi: 10.1523/JNEUROSCI.4478-10.2010

Deshar, R., Moon, S., Yoo, W., Cho, E. B., Yoon, S. K., and Yoon, J. B. (2016). RNF167 targets Arl8B for degradation to regulate lysosome positioning and endocytic trafficking. FEBS J. 283, 4583-4599. doi: 10.1111/febs. 13947

Dibbens, L. M., Ekberg, J., Taylor, I., Hodgson, B. L., Conroy, S. J., Lensink, I. L., et al. (2007). NEDD4-2 as a potential candidate susceptibility gene for epileptic photosensitivity. Genes Brain Behav. 6, 750-755. doi: 10.1111/j.1601-183x. 2007.00305.x

Diering, G. H., Nirujogi, R. S., Roth, R. H., Worley, P. F., Pandey, A., and Huganir, R. L. (2017). Homerla drives homeostatic scaling-down of excitatory synapses during sleep. Science 355, 511-515. doi: 10.1126/science. aai8355

Ehlers, M. D. (2000). Reinsertion or degradation of AMPA receptors determined by activity-dependent endocytic sorting. Neuron 28, 511-525. doi: 10.1016/s0896-6273(00)00129-x

Epi4K Consortium, Epilepsy Phenome/Genome Project, Allen, A. S., Berkovic, S. F., Cossette, P., Delanty, N., et al. (2013). De novo mutations in epileptic encephalopathies. Nature 501, 217-221. doi: 10.1038/nature12439

Fernández-Monreal, M., Brown, T. C., Royo, M., and Esteban, J. A. (2012). The balance between receptor recycling and trafficking toward lysosomes determines synaptic strength during long-term depression. J. Neurosci. 32, 13200-13205. doi: 10.1523/JNEUROSCI.0061-12.2012

\section{AUTHOR CONTRIBUTIONS}

JW, SG, SEJ and VA wrote, revised and finalized this manuscript.

\section{ACKNOWLEDGMENTS}

This work was supported by grants from the John T. Reid Charitable Trusts, the Alzheimer's Australia Dementia Research Foundation (DGP14-57), the Australian National Health and Medical Research Council (GNT1083209 and GNT1099114), the Australian Research Council (ARC, DP170102402) and the Clem Jones Centre for Ageing Dementia Research to VA. JW is an ARC DECRA Fellow (DE170100112). SG and SEJ are recipients of University of Queensland International Scholarships. We thank Rowan Tweedale for editing of this manuscript.

French, J. A., Krauss, G. L., Biton, V., Squillacote, D., Yang, H., Laurenza, A., et al. (2012). Adjunctive perampanel for refractory partial-onset seizures: randomized phase III study 304. Neurology 79, 589-596. doi: 10.1212/WNL. 0b013e3182635735

French, J. A., Krauss, G. L., Wechsler, R. T., Wang, X. F., DiVentura, B., Brandt, C., et al. (2015). Perampanel for tonic-clonic seizures in idiopathic generalized epilepsy A randomized trial. Neurology 85, 950-957. doi: 10.1212/WNL. 0000000000001930

Fu, A. K., Hung, K. W., Fu, W. Y., Shen, C., Chen, Y., Xia, J., et al. (2011). $\mathrm{APC}^{\mathrm{Cdh} 1}$ mediates EphA4-dependent downregulation of AMPA receptors in homeostatic plasticity. Nat. Neurosci. 14, 181-189. doi: 10.1038/nn.2715

Goo, M. S., Scudder, S. L., and Patrick, G. N. (2015). Ubiquitin-dependent trafficking and turnover of ionotropic glutamate receptors. Front. Mol. Neurosci. 8:60. doi: 10.3389/fnmol.2015.00060

Govers, R., ten Broeke, T., van Kerkhof, P., Schwartz, A. L., and Strous, G. J. (1999). Identification of a novel ubiquitin conjugation motif, required for ligandinduced internalization of the growth hormone receptor. EMBO J. 18, 28-36. doi: 10.1093/emboj/18.1.28

Guntupalli, S., Jang, S. E., Zhu, T., Huganir, R. L., Widagdo, J., and Anggono, V. (2017). GluA1 subunit ubiquitination mediates amyloid- $\beta$-induced loss of surface $\alpha$-amino-3-hydroxy-5-methyl-4-isoxazolepropionic acid (AMPA) receptors. J. Biol. Chem. 292, 8186-8194. doi: 10.1074/jbc.M116.774554

Guntupalli, S., Widagdo, J., and Anggono, V. (2016). Amyloid- $\beta$-induced dysregulation of AMPA receptor trafficking. Neural Plast. 2016:3204519. doi: $10.1155 / 2016 / 3204519$

Harper, J. W., Burton, J. L., and Solomon, M. J. (2002). The anaphase-promoting complex: it's not just for mitosis any more. Genes Dev. 16, 2179-2206. doi: $10.1101 / \operatorname{gad} .1013102$

Haugsten, E. M., Malecki, J., Bjorklund, S. M., Olsnes, S., and Wesche, J. (2008). Ubiquitination of fibroblast growth factor receptor 1 is required for its intracellular sorting but not for its endocytosis. Mol. Biol. Cell 19, 3390-3403. doi: 10.1091/mbc.E07-12-1219

Hayashi, T., Thomas, G. M., and Huganir, R. L. (2009). Dual palmitoylation of NR2 subunits regulates NMDA receptor trafficking. Neuron 64, 213-226. doi: 10.1016/j.neuron.2009.08.017

Hershko, A., and Ciechanover, A. (1998). The ubiquitin system. Annu. Rev. Biochem. 67, 425-479. doi: 10.1146/annurev.biochem.67.1.425

Hill, T. A., Gordon, C. P., McGeachie, A. B., Venn-Brown, B., Odell, L. R., Chau, N., et al. (2009). Inhibition of dynamin mediated endocytosis by the dynoles-synthesis and functional activity of a family of indoles. J. Med. Chem. 52, 3762-3773. doi: 10.1021/jm900036m

Hou, Q., Gilbert, J., and Man, H. Y. (2011). Homeostatic regulation of AMPA receptor trafficking and degradation by light-controlled single-synaptic activation. Neuron 72, 806-818. doi: 10.1016/j.neuron.2011.10.011

Huang, J., and Bonni, A. (2016). A decade of the anaphase-promoting complex in the nervous system. Genes Dev. 30, 622-638. doi: 10.1101/gad.274324.115 
Huang, X., and Dixit, V. M. (2016). Drugging the undruggables: exploring the ubiquitin system for drug development. Cell Res. 26, 484-498. doi: 10.1038/cr. 2016.31

Huang, F., Goh, L. K., and Sorkin, A. (2007). EGF receptor ubiquitination is not necessary for its internalization. Proc. Natl. Acad. Sci. U S A 104, 16904-16909. doi: 10.1073/pnas.0707416104

Huang, J., Ikeuchi, Y., Malumbres, M., and Bonni, A. (2015). A Cdh1-APC/FMRP ubiquitin signaling link drives mGluR-dependent synaptic plasticity in the mammalian brain. Neuron 86, 726-739. doi: 10.1016/j.neuron.2015. 03.049

Huganir, R. L., and Nicoll, R. A. (2013). AMPARs and synaptic plasticity: the last 25 years. Neuron 80, 704-717. doi: 10.1016/j.neuron.2013.10.025

Huo, Y., Khatri, N., Hou, Q., Gilbert, J., Wang, G., and Man, H. Y. (2015). The deubiquitinating enzyme USP46 regulates AMPA receptor ubiquitination and trafficking. J. Neurochem. 134, 1067-1080. doi: 10.1111/jnc.13194

Ikeda, F., and Dikic, I. (2008). Atypical ubiquitin chains: new molecular signals. 'Protein modifications: beyond the usual suspects' review series. EMBO Rep. 9, 536-542. doi: 10.1038/embor.2008.93

Jewett, K. A., Zhu, J., and Tsai, N. P. (2015). The tumor suppressor p53 guides GluA1 homeostasis through Nedd4-2 during chronic elevation of neuronal activity. J. Neurochem. 135, 226-233. doi: 10.1111/jnc.13271

Juo, P., and Kaplan, J. M. (2004). The anaphase-promoting complex regulates the abundance of GLR-1 glutamate receptors in the ventral nerve cord of C. elegans. Curr. Biol. 14, 2057-2062. doi: 10.1016/j.cub.2004. 11.010

Kim, H. T., Kim, K. P., Lledias, F., Kisselev, A. F., Scaglione, K. M., Skowyra, D., et al. (2007). Certain pairs of ubiquitin-conjugating enzymes (E2s) and ubiquitin-protein ligases (E3s) synthesize nondegradable forked ubiquitin chains containing all possible isopeptide linkages. J. Biol. Chem. 282, 17375-17386. doi: 10.1074/jbc.M609659200

Komander, D., and Rape, M. (2012). The ubiquitin code. Annu. Rev. Biochem. 81, 203-229. doi: 10.1146/annurev-biochem-060310-170328

Kowalski, J. R., Dahlberg, C. L., and Juo, P. (2011). The deubiquitinating enzyme USP-46 negatively regulates the degradation of glutamate receptors to control their abundance in the ventral nerve cord of Caenorhabditis elegans. J. Neurosci. 31, 1341-1354. doi: 10.1523/jneurosci.4765-10.2011

Lee, J.-A., and Gao, F.-B. (2012). Neuronal functions of ESCRTs. Exp. Neurobiol. 21, 9-15. doi: 10.5607/en.2012.21.1.9

Lin, A., Hou, Q., Jarzylo, L., Amato, S., Gilbert, J., Shang, F., et al. (2011). Nedd4mediated AMPA receptor ubiquitination regulates receptor turnover and trafficking. J. Neurochem. 119, 27-39. doi: 10.1111/j.1471-4159.2011.07221.x

Lin, J. W., Ju, W., Foster, K., Lee, S. H., Ahmadian, G., Wyszynski, M., et al. (2000). Distinct molecular mechanisms and divergent endocytotic pathways of AMPA receptor internalization. Nat. Neurosci. 3, 1282-1290. doi: 10.1038/81814

Lin, D.-T., Makino, Y., Sharma, K., Hayashi, T., Neve, R., Takamiya, K., et al. (2009). Regulation of AMPA receptor extrasynaptic insertion by $4.1 \mathrm{~N}$, phosphorylation and palmitoylation. Nat. Neurosci. 12, 879-887. doi: $10.1038 / \mathrm{nn} .2351$

Lin, A., and Man, H. Y. (2014). Endocytic adaptor epidermal growth factor receptor substrate 15 (Eps15) is involved in the trafficking of ubiquitinated $\alpha$-amino-3-hydroxy-5-methyl-4-isoxazolepropionic acid receptors. J. Biol. Chem. 289, 24652-24664. doi: 10.1074/jbc.m114.582114

$\mathrm{Lu}, \mathrm{W}$., and Roche, K. W. (2012). Posttranslational regulation of AMPA receptor trafficking and function. Curr. Opin. Neurobiol. 22, 470-479. doi: 10.1016/j. conb.2011.09.008

Lussier, M. P., Herring, B. E., Nasu-Nishimura, Y., Neutzner, A., Karbowski, M., Youle, R. J., et al. (2012). Ubiquitin ligase RNF167 regulates AMPA receptormediated synaptic transmission. Proc. Natl. Acad. Sci. U S A 109, 19426-19431. doi: 10.1073/pnas.1217477109

Lussier, M. P., Nasu-Nishimura, Y., and Roche, K. W. (2011). Activity-dependent ubiquitination of the AMPA receptor subunit GluA2. J. Neurosci. 31, 3077-3081. doi: 10.1523/jneurosci.5944-10.2011

Mabb, A. M., and Ehlers, M. D. (2010). Ubiquitination in postsynaptic function and plasticity. Annu. Rev. Cell Dev. Biol. 26, 179-210. doi: 10.1146/annurevcellbio-100109-104129

Macia, E., Ehrlich, M., Massol, R., Boucrot, E., Brunner, C., and Kirchhausen, T. (2006). Dynasore, a cell-permeable inhibitor of dynamin. Dev. Cell 10, 839-850. doi: 10.1016/j.devcel.2006.04.002
O’Brien, R. J., Kamboj, S., Ehlers, M. D., Rosen, K. R., Fischbach, G. D., and Huganir, R. L. (1998). Activity-dependent modulation of synaptic AMPA receptor accumulation. Neuron 21, 1067-1078. doi: 10.1016/s08966273(00)80624-8

Patrick, G. N., Bingol, B., Weld, H. A., and Schuman, E. M. (2003). Ubiquitinmediated proteasome activity is required for agonist-induced endocytosis of GluRs. Curr. Biol. 13, 2073-2081. doi: 10.1016/j.cub.2003.10.028

Persaud, A., Alberts, P., Amsen, E. M., Xiong, X., Wasmuth, J., Saadon, Z., et al. (2009). Comparison of substrate specificity of the ubiquitin ligases Nedd 4 and Nedd4-2 using proteome arrays. Mol. Syst. Biol. 5:333. doi: 10.1038/msb. 2009.85

Plant, P. J., Yeger, H., Staub, O., Howard, P., and Rotin, D. (1997). The C2 domain of the ubiquitin protein ligase Nedd4 mediates $\mathrm{Ca}^{2+}$-dependent plasma membrane localization. J. Biol. Chem. 272, 32329-32336. doi: 10.1074/jbc.272. 51.32329

Popoli, M., Yan, Z., McEwen, B. S., and Sanacora, G. (2012). The stressed synapse: the impact of stress and glucocorticoids on glutamate transmission. Nat. Rev. Neurosci. 13, 22-37. doi: $10.1038 / \mathrm{nrn} 3138$

Pozo, K., and Goda, Y. (2010). Unraveling mechanisms of homeostatic synaptic plasticity. Neuron 66, 337-351. doi: 10.1016/j.neuron.2010. 04.028

Roche, K. W., O’Brien, R. J., Mammen, A. L., Bernhardt, J., and Huganir, R. L. (1996). Characterization of multiple phosphorylation sites on the AMPA receptor GluR1 subunit. Neuron 16, 1179-1188. doi: 10.1016/s08966273(00)80144-0

Rodrigues, E. M., Scudder, S. L., Goo, M. S., and Patrick, G. N. (2016). A $\beta$-induced synaptic alterations require the E3 ubiquitin ligase Nedd4-1. J. Neurosci. 36, 1590-1595. doi: 10.1523/JNEUROSCI.2964-15.2016

Rogawski, M. A. (2013). AMPA receptors as a molecular target in epilepsy therapy. Acta Neurol. Scand. Suppl. 197, 9-18. doi: 10.1111/ane. 12099

Scheffner, M., and Kumar, S. (2014). Mammalian HECT ubiquitin-protein ligases: biological and pathophysiological aspects. Biochim. Biophys. Acta 1843, 61-74. doi: 10.1016/j.bbamcr.2013.03.024

Schwarz, L. A., Hall, B. J., and Patrick, G. N. (2010). Activity-dependent ubiquitination of GluA1 mediates a distinct AMPA receptor endocytosis and sorting pathway. J. Neurosci. 30, 16718-16729. doi: 10.1523/jneurosci.368610.2010

Scudder, S. L., Goo, M. S., Cartier, A. E., Molteni, A., Schwarz, L. A., Wright, R., et al. (2014). Synaptic strength is bidirectionally controlled by opposing activity-dependent regulation of Nedd4-1 and USP8. J. Neurosci. 34, 16637-16649. doi: 10.1523/JNEUROSCI.2452-14.2014

Selkoe, D. J. (2002). Alzheimer's disease is a synaptic failure. Science 298, 789-791. doi: 10.1126/science.1074069

Selvakumar, B., Jenkins, M. A., Hussain, N. K., Huganir, R. L., Traynelis, S. F., and Snyder, S. H. (2013). S-nitrosylation of AMPA receptor GluAl regulates phosphorylation, single-channel conductance, and endocytosis. Proc. Natl. Acad. Sci. U S A 110, 1077-1082. doi: 10.1073/pnas.1221295110

Sheng, M., Sabatini, B. L., and Sudhof, T. C. (2012). Synapses and Alzheimer's disease. Cold Spring Harb. Perspect. Biol. 4:a005777. doi: 10.1101/cshperspect. a005777

Sowa, M. E., Bennett, E. J., Gygi, S. P., and Harper, J. W. (2009). Defining the human deubiquitinating enzyme interaction landscape. Cell 138, 389-403. doi: 10.1016/j.cell.2009.04.042

Sukumaran, M., Penn, A. C., and Greger, I. H. (2012). AMPA receptor assembly: atomic determinants and built-in modulators. Adv. Exp. Med. Biol. 970, 241-264. doi: 10.1007/978-3-7091-0932-8_11

Terashima, A., Pelkey, K. A., Rah, J. C., Suh, Y. H., Roche, K. W., Collingridge, G. L., et al. (2008). An essential role for PICK1 in NMDA receptor-dependent bidirectional synaptic plasticity. Neuron 57, 872-882. doi: $10.1016 /$ j.neuron.2008.01.028

Thorsen, T. S., Madsen, K. L., Rebola, N., Rathje, M., Anggono, V., Bach, A., et al. (2010). Identification of a small-molecule inhibitor of the PICK1 PDZ domain that inhibits hippocampal LTP and LTD. Proc. Natl. Acad. Sci. U S A 107, 413-418. doi: 10.1073/pnas.0902225107

Turrigiano, G. (2012). Homeostatic synaptic plasticity: local and global mechanisms for stabilizing neuronal function. Cold Spring Harb. Perspect. Biol. 4:a005736. doi: 10.1101/cshperspect.a005736 
Turrigiano, G. G., Leslie, K. R., Desai, N. S., Rutherford, L. C., and Nelson, S. B. (1998). Activity-dependent scaling of quantal amplitude in neocortical neurons. Nature 391, 892-896. doi: 10.1038/ 36103

van Dijk, J. R., Yamazaki, Y., and Palmer, R. H. (2014). Tumour-associated mutations of PA-TM-RING ubiquitin ligases RNF167/RNF13 identify the PA domain as a determinant for endosomal localization. Biochem. J. 459, 27-36. doi: 10.1042/bj20131067

Vanli-Yavuz, E. N., Ozdemir, O., Demirkan, A., Catal, S., Bebek, N., Ozbek, U., et al. (2015). Investigation of the possible association of NEDD4-2 (NEDD4L) gene with idiopathic photosensitive epilepsy. Acta Neurol. Belg. 115, 241-245. doi: $10.1007 /$ s13760-014-0412-x

Widagdo, J., and Anggono, V. (2015). Ubiquitin signals the demise of AMPA receptors. Oncotarget 6, 15718-15719. doi: 10.18632/oncotarget.4645

Widagdo, J., Chai, Y. J., Ridder, M. C., Chau, Y. Q., Johnson, R. C., Sah, P., et al. (2015). Activity-dependent ubiquitination of GluA1 and GluA2 regulates AMPA receptor intracellular sorting and degradation. Cell Rep. 10, 783-795. doi: 10.1016/j.celrep.2015.01.015

Widagdo, J., Fang, H., Jang, S. E., and Anggono, V. (2016). PACSIN1 regulates the dynamics of AMPA receptor trafficking. Sci. Rep. 6:31070. doi: $10.1038 /$ srep31070

Willem, M., Tahirovic, S., Busche, M. A., Ovsepian, S. V., Chafai, M., Kootar, S., et al. (2015). $\eta$-secretase processing of APP inhibits neuronal activity in the hippocampus. Nature 526, 443-447. doi: 10.1038/nature 14864

Wright, M. H., Berlin, I., and Nash, P. D. (2011). Regulation of endocytic sorting by ESCRT-DUB-mediated deubiquitination. Cell Biochem. Biophys. 60, 39-46. doi: 10.1007/s12013-011-9181-9

Xia, J., Zhang, X., Staudinger, J., and Huganir, R. L. (1999). Clustering of AMPA receptors by the synaptic PDZ domain-containing protein PICK1. Neuron 22, 179-187. doi: 10.1016/s0896-6273(00)80689-3
Yamazaki, Y., Schönherr, C., Varshney, G. K., Dogru, M., Hallberg, B., and Palmer, R. H. (2013). Goliath family E3 ligases regulate the recycling endosome pathway via VAMP3 ubiquitylation. EMBO J. 32, 524-537. doi: 10.1038/emboj. 2013.1

Yang, B., and Kumar, S. (2010). Nedd4 and Nedd4-2: closely related ubiquitinprotein ligases with distinct physiological functions. Cell Death Differ. 17, 68-77. doi: 10.1038/cdd.2009.84

Yuen, E. Y., Wei, J., Liu, W., Zhong, P., Li, X., and Yan, Z. (2012). Repeated stress causes cognitive impairment by suppressing glutamate receptor expression and function in prefrontal cortex. Neuron 73, 962-977. doi: 10.1016/j.neuron.2011. 12.033

Zhang, D., Hou, Q., Wang, M., Lin, A., Jarzylo, L., Navis, A., et al. (2009). $\mathrm{Na}, \mathrm{K}-\mathrm{ATP}$ ase activity regulates AMPA receptor turnover through proteasomemediated proteolysis. J. Neurosci. 29, 4498-4511. doi: 10.1523/jneurosci.609408.2009

Zhu, J., Lee, K. Y., Jewett, K. A., Man, H. Y., Chung, H. J., and Tsai, N. P. (2017). Epilepsy-associated gene Nedd4-2 mediates neuronal activity and seizure susceptibility through AMPA receptors. PLoS Genet. 13:e1006634. doi: 10.1371/journal.pgen.1006634

Conflict of Interest Statement: The authors declare that the research was conducted in the absence of any commercial or financial relationships that could be construed as a potential conflict of interest.

Copyright (c) 2017 Widagdo, Guntupalli, Jang and Anggono. This is an open-access article distributed under the terms of the Creative Commons Attribution License (CC BY). The use, distribution or reproduction in other forums is permitted, provided the original author(s) or licensor are credited and that the original publication in this journal is cited, in accordance with accepted academic practice. No use, distribution or reproduction is permitted which does not comply with these terms. 